ESJ Humanities

\title{
El Capital Intelectual y su Incidencia en la Producción de las Empresas Productoras de Banano en la Costa sur del Ecuador
}

\author{
Iddar Iván Jaya Pineda \\ Jorge Javier Plaza Guzmán \\ Ernesto Novillo Maldonado
}

Docente Universidad Técnica de Machala, Ecuador

\section{Doi:10.19044/esj.2021.v17n9p17}

Submitted: 07 November 2020

Accepted: 12 February 2021

Published: 31 March 2021
Copyright 2021 Author(s)

Under Creative Commons BY-NC-ND

4.0 OPEN ACCESS

Cite As:

Jaya Pineda I.I., Plaza Guzmán J.J. \& Novillo Maldonado E. (2021). El Capital Intelectual y su Incidencia en la Producción de las Empresas Productoras de Banano en la Costa sur del Ecuador. European Scientific Journal, ESJ, 17(9), 17.

https://doi.org/10.19044/esj.2021.v17n9p17

\section{Resumen}

El presente trabajo de investigación se lo realizó por el entorno extremadamente competitivo en el área agrícola exigiendo a las empresas productoras de banano un esfuerzo constante en la mejora de sus procesos de producción, comercialización, gestión y dirección económica con un capital de talento humano calificado en el área de siembra y administración de las fincas. De esta manera los pequeños productores de banano tradicional se han visto obligados a depender cada vez más de la información técnica en el área agrícola, administrativa financiera proporcionada por un capital intelectual humano de alto nivel que toma como base objetiva ejercer una función vital: la toma de decisiones, donde se aprecia que las agrícolas de economía familiar carecen de un capital intelectual idóneo para ejercer esta función, a diferencia de las fincas asociadas que su visión está orientando para insertarse y permanecer en el mercado bananero con productos de calidad, con volúmenes y periodicidad demandada, que permitan el desarrollo sostenible de la organización, en el mercado interno como internacional, dando prioridad en los estándar de calidad en la fruta de exportación.

Palabras claves: Competitividad, humano, producción, rentabilidad 


\title{
Intellectual Capital and its Impact on the Production of Small Banana-Producing Companies in the Southern Coast of Ecuador
}

\author{
Iddar Iván Jaya Pineda \\ Jorge Javier Plaza Guzmán \\ Ernesto Novillo Maldonado \\ Docente Universidad Técnica de Machala, Ecuador
}

\begin{abstract}
This paper was carried out due to the extremely competitive environment in the agricultural area. This requires the banana-producing companies to make a constant effort to improve their production, marketing, management, and economic management processes with a capital of qualified human talent in the area of sowing and administration of the farms. Through this way, small traditional banana producers have been forced to rely more and more on technical information in the agricultural, administrative, and financial area provided by a high-level human intellectual capital. This gave rise to the objective of performing a vital function, which is decision-making. Here, it can be seen that family farmers lack the ideal intellectual capital to exercise this function. This is unlike the associated farms where their vision is guiding to insert and remain in the banana market with quality products, with volumes and demanded periodicity. This has also led to the sustainable development of the organization, in the domestic and international markets, by giving priority to quality standards in export fruit.
\end{abstract}

Keywords: Competitiveness, human, production, profitability

\section{Introduction}

La investigación se presenta como un diagnóstico y una alternativa socioeconómica sostenible del capital intelectual en las empresas productoras de banano en la provincia de El Oro, en la década de los 50 hasta el presente, la producción bananera es el impulso de la economía ecuatoriana, apoderándose de la exportación no petrolera del país, esta actividad agrícola influye directamente en la dinamización monetaria en las arcas del estado. Como generador de divisas y en forma directa en la generación de empleo y favoreciendo el crecimiento de otras actividades (Minsterio de Agricultura,ganaderia,acualcultura y pesca, 2019).

La siembra, producción y comercialización bananera, es creadora de empleos del 17\% de la población económicamente activa. En Ecuador, 4 de 
cada 10 personas de la zona rural son pobres y 7 de cada 10 empleos provienen del sector agropecuario (Arbeláez, 2019). El valor agregado que va mucho más allá de su propia actividad, es decir una unidad de siembra de banano da origen a una serie de otras actividades económicas y, viceversa, una menos puede llevar a una involución en las otras (Viteri, 2018).

Las excelentes condiciones geográficas, climáticas y ecológicas que posee el Ecuador, favorecen al desarrollo de la explotación de banano que "lo reconoce como la fruta perfecta, tanto por su sabor, textura, dulzura y duración en percha, como por el impacto socioeconómico que tiene su producción y comercialización" (Ministerio de Agricultura y Ganaderia, 2019).

En la provincia de El Oro el 30\% del movimiento económico laboral corresponden de manera directa a las fincas bananeras del sector (Carrrillo, 2018). Además, en esta región contempla el mayor asentamiento de pequeños y medianos productores de cultivo de banano a escala nacional, según datos de la subsecretaria de comercialización del Ministerio de Agricultura, es decir de los 5.737 productores registrados a nivel país, 2.375 el 41,39 \% pertenecen a esta provincia orense, radicándose en los cantones de Machala, Pasaje, Santa Rosa, Arenillas y El Guabo. Esta actividad de cultivo de banano es desarrollada mayoritariamente por quienes han heredado el negocio, por ende, el desarrollo de sus actividades económicas se realiza de manera empírica y tradicional (Gaibor, 2018).

Dando como resultado en la producción y en la administración marcadas limitaciones, generando incertidumbre y desorientación en la comprensión del resultado económico y retraso en la toma de decisiones (Instituto de Investigaciones Económicas y Políticas (IIEP), 2017). De esta manera resulta de vital importancia en el análisis la parte del capital humano de las empresas agrícolas donde pasan de ser un factor meramente instrumental a constituirse en un factor estratégico y clave en el desarrollo de las empresas, tomando como protagonistas al campo laboral (empleados, trabajadores) para el logro de los objetivos propuestos.

\section{Análisis de la demanda del banano ecuatoriano en el mercado internacional}

Ecuador se permite abastecer la demanda mundial los 365 días del año, aproximadamente el $90 \%$ de la producción regional es aportada por el perfil costanero del Ecuador y los principales mercados son Rusia y la Unión Europea (UE) (Acorbanec, 2019).

Desde hace tres décadas, Ecuador es líder en la exportación de banano, en la actualidad representa el $26 \%$ de las exportaciones mundiales. Sus principales competidores a nivel mundial en el mercado bananero son: Filipinas, Guatemala, Costa Rica, Colombia, India, China, Brasil, convirtiendo a América como el continente más importante de producción 
(Jaramillo et al., 2020). El $81 \%$ del consumo de esta fruta en el globo terrestre proviene de estos 10 países que se indica en de la siguiente ilustración.

Gráfico 1. LOS 10 PRINCIPALES PAISES EXPORTADORES DE BANANO

PARTICIPACION DE EXPORTACIONES MUNDIALES DE BANANO

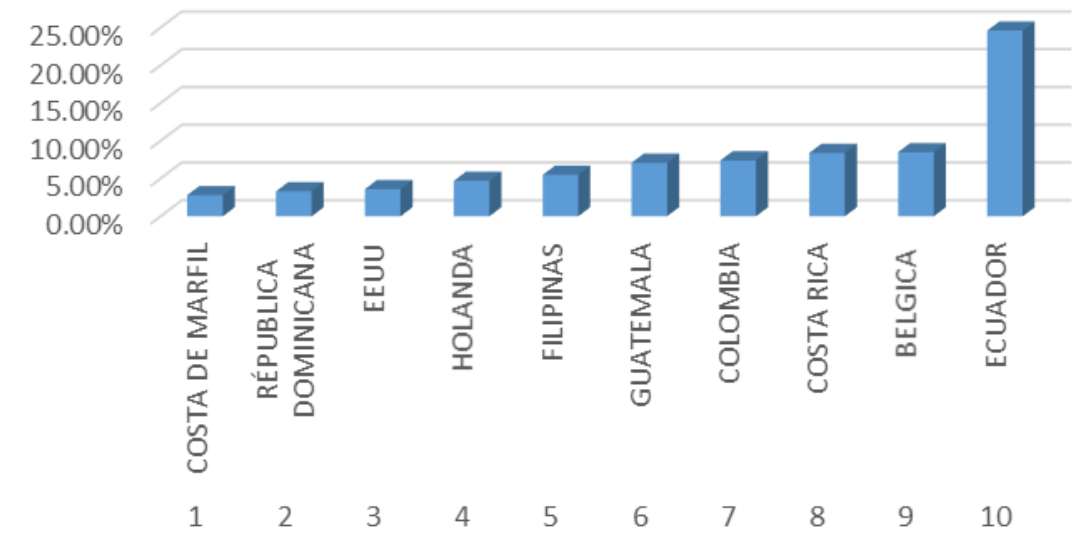

Fuente: Banano Emizor

Elaborado: Autoría propia

Ecuador se sitúa en 1er lugar en exportaciones de banano del mundo, de hecho, 1 de cada 4 bananos que se consumen en el planeta son producidos en territorio ecuatoriano. La industria bananera se consolida con las grandes empresas predominantes en las exportaciones de banano del mundo (Zabala, 2019).

En la presente grafica se expone proyectado en miles (dólares) desde el año 1900 una tendencia a la alza con una acertada aceptación de la fruta en el mercado internacional, estudio hasta el año 2018 (Numbersnews, 2019). 
Grafico 2.

\section{Exportaciones Banano-Ecuador miles USD}

Crecimiento de las exportaciones desde el año 1990 al 2018

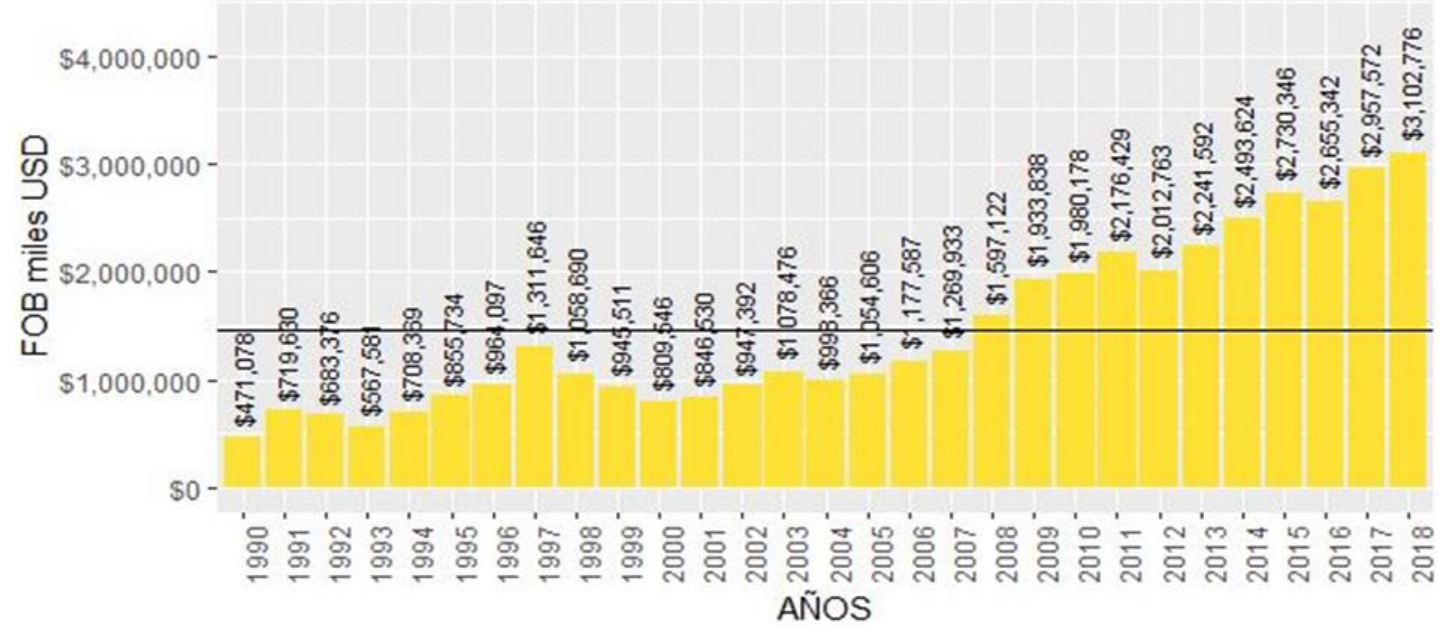

Fuente: Banco Central del Ecuador

Elaborado: (Numbersnews, 2019)

Las exportaciones bananeras de enero a noviembre del 2019 fueron superiores al 2018, con un incremento del $1.65 \%$ en los envió de cajas de banano al exterior, a pesar que sufrió un bajón el tercer trimestre (julio, agosto, septiembre) del 2019 (La Asociación de Exportadores de Banano de Ecuador (AEBE), 2020). Influenciaron las situaciones climatológicas (bajas temperaturas), como la paralización protagonizada por algunos sectores y movimientos sociales en este periodo, obligaron a no poder embarcar mayor cantidad de fruta. En este escenario hasta finales de noviembre, se enviaron 322,7 millones de cajas de 43 libras, mientras que en el 2018 fueron 317,4 millones (El Comercio, 2020).

En 2020 las cifras son halagadoras considerando el impacto por la crisis sanitaria, social y económica producida por la pandemia del coronavirus (COVID-19), toda la cadena de producción y abastecimiento sufrió serios daños colaterales ( El Foro Mundial Bananero, 2020).

\section{Incidencia económica de la exportación bananera en Ecuador en el año 2020}

A pesar de la crisis sanitaria hay un despunte "para el año 2020, la economía ecuatoriana recuperará paulatinamente la senda de crecimiento al prever una tasa de variación de 0,69\%, alcanzando un PIB constante de USD 72.309 millones y un PIB nominal de USD 109.667 millones, mayor al del año anterior 2019" (Banco Central del Ecuador, 2020). 


\section{Gráfico 3. PRODUCTO INTERNO BRUTO (PIB)}

USD millones, $2007=100$

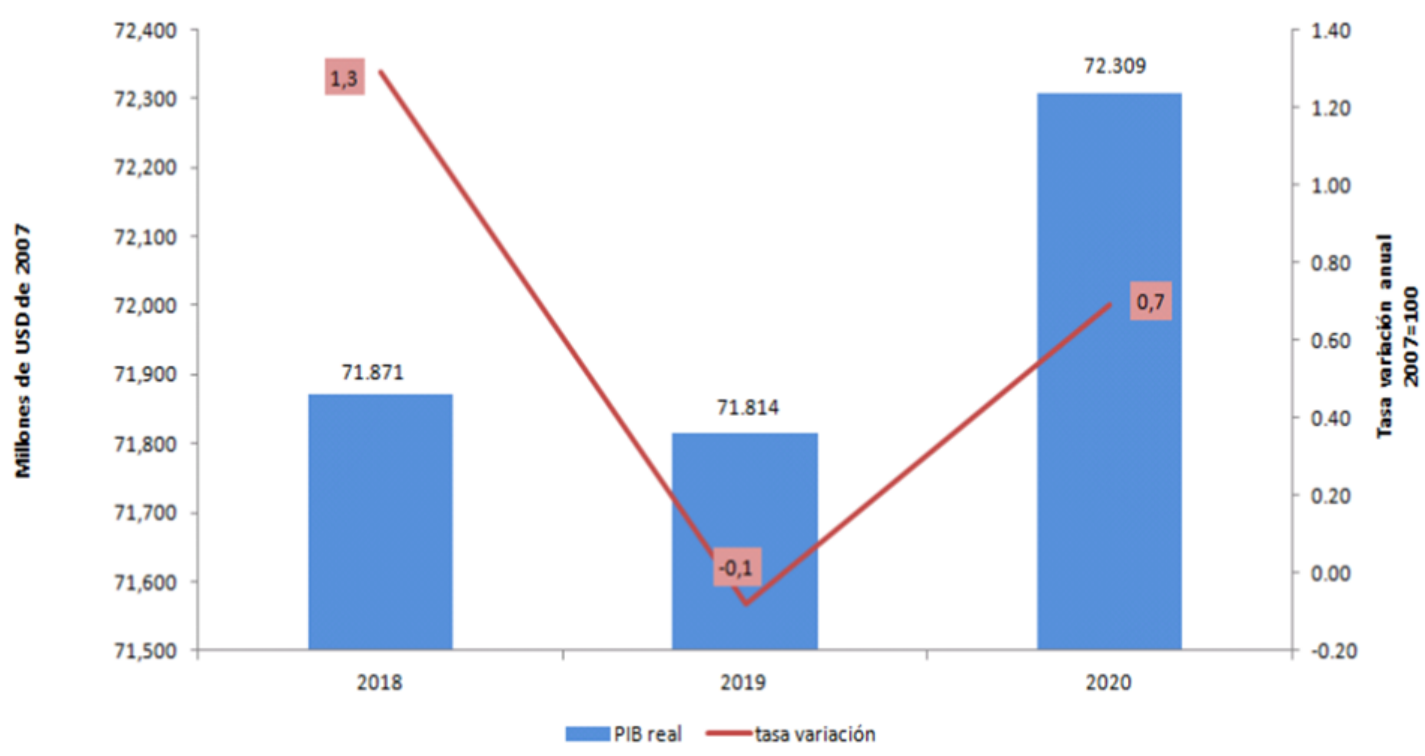

Fuente: Banco Central del Ecuador

Elaborado: (Banco Central del Ecuador, 2020)

\section{Análisis local y su problemática de las agrícolas bananeras}

En el área de estudio de las costas sur del Ecuador geográficamente sitúa a la Provincia de el Oro, reconocida cabecera cantonal Machala como la capital bananera del mundo (León \& Martínez, 2012). Donde la plantación de banano se centra en los cantones de Machala, Pasaje, Santa Rosa, Arenillas y El Guabo (Ollague et al., 2019). Un mínimo porcentaje tienen una asistencia técnica con profesionales de la región con experiencia en el proceso de cultivo, cosecha, empaque, comercialización de la fruta, como en el aérea administrativa y contable, obteniendo resultados óptimos en la producción de sus plantaciones (Instituto Nacional de Investigaciones Agropecuaria, 2016). Este es el resultado que se han agrupado en asociaciones minimizando los problemas fitosanitarios en control de plagas utilizando productos amigables con el ambiente, responsabilidad social, maximizando la utilización de sus tierras como en la parte financiera, estimulados por una economía popular y solidaria (Arguello et al., 2019)

Referente a los pequeños agricultores se encuentran rezagados y este sector agrícola viene perdiendo dinamismo en consecuencia, que aún mantienen de manera empírica el sistema de producción y de gestión en sus parcelas, se identifica a este grupo de agricultores, como de la economía familiar, donde de manera acelerada va perdiendo espacio en el mercado 
bananero (Revista Lideres, 2019). Se considera los empleos que generan son informales, lo que permite que los ingresos de colaboradores de las fincas, sea en general precario (Ministerio de Comercio Exterior, 2017).

\section{Características de las finca productoras de banano de la provincia de El Oro}

- En el estudio de las agrícolas bananeras se detecta algunas características:

- Unidades de producción familiares, que pasan de generación en generación.

- Subdivisión de tierra entre herederos que resulta en extensiones no representativas de una unidad económica.

- Predisposición en la aplicación de tecnologías de producción.

- No adopta herramientas de cobertura de precio o riesgos climáticos para las plantaciones, básicamente por desconocimiento de las mismas.

- Las agrícolas acostumbradas a reprogramaciones y refinanciación de deudas en las entidades financieras.

\section{El capital humano en las fincas de producción de banano de la localidad}

Los mercados bananeros se tornan más exigentes y por esta razón los intangibles se vuelven factores para el éxito del presente y futuro de las empresas. La gestión desde el punto de vista empresarial tiene como objetivo primordial, obtener resultados positivos para la organización en el área de la productividad, rentabilidad, satisfacción en el talento humano y responsabilidad corporativa (Pérez, 2016).

Frente a la problemática antes citada con las agrícolas bananeras y sus características ante los cambios tecnológicos y sociales, que exige la competitividad se presentan cambios en el método de producción (siembra, cosecha, empaque) hasta la comercialización, donde es pertinente un proceso de selección del talento humano, se presente en función de las necesidades de las fincas productoras (Fajardo, 2015).

La gestión de los recursos humanos ha tenido un progreso desde la tradicional administración de personal hasta la gestión del conocimiento en desarrollo, proceso que según Cuesta (2005), ha comprendido esencialmente de tres paradigmas: los recursos humanos como costo, como activo e inversor de su potencial humano o capital humano (Sablich, 2012).

Drucker (2002) afirma para que esto se cumpla interviene cuatro factores. La estrategia, que significa un conjunto de acciones para llegar a una meta. La cultura, donde se sensibiliza los valores y el compromiso ético de la organización. La Estructura, la manera organizada en infraestructura y en personas calificadas. La toma de decisiones, escoger las medidas necesarias 
con un compromiso institucional. Esta nueva mentalidad de negocios permite que las organizaciones busquen su identidad destacada para distinguirse de las demás, para situarse dentro de su población objetivo (Monje, 2011).

En el país el constante esfuerzo de las clases sociales por una estabilidad económica, y equidad en el reparto de la riqueza, permite tener una estrategia de contar con personal calificado en todas las áreas del proceso agrícola, con fines que se vuelva una producción sustentable para los agricultores (Coraggio, 2011).

Razón por la cual resulta menester recurrir al capital humano como un sistema adaptado para clasificar los hechos de cultivo, administrativos y financieros (Soler, 2017). Es de vital importancia mantener y perfeccionar el proceso de producción que ocurre en las fincas de banano (Pardo \& Novillo, 2016). De tal manera que, se convierte en el eje central para llevar a cabo diversos procedimientos que conducirán a la obtención del máximo rendimiento productivo y monetario en la empresa bananera (Heredia, 2018).

\section{Modalidad aplicada}

La presente investigación se utilizó en la modalidad de trabajo experimental, debido ser un trabajo de encuestas no se aplicó análisis bioestadística, sino estadístico inferencial.

\section{Tipo de investigación: Descriptivo-estadístico}

Para su estructuración y aplicación se utilizó procedimientos y técnicas basadas en el método descriptivo. La investigación se dio de carácter cuantitativa, la recolección de la información se efectuará mediante encuestas personales a los productores o administradores de las fincas.

\section{Métodos, técnicas e instrumentos Métodos}

Se utilizarán los métodos de la Investigación de Campo, que consiste en el análisis sistemático de problemas de la realidad, con el propósito bien sea de describirlos, interpretarlos entender su naturaleza y factores constituyentes.

\section{Técnicas}

Etapa 1: Fue definida e implementada una propuesta de categorización de las haciendas muestreadas, tomando como referencia el listado de haciendas que se encuentran en el área de estudio con lo cual se delimita el área de influencia y sus características.

Etapa 2: Se realizó un diagnóstico general que incluye indicadores básicos de la implementación del capital intelectual humano en áreas del campo, manejo en la plantación, en el área de empaque y administración con 
la que cuenta las fincas bananeras, para lo cual se utilizó la técnica de la encuesta, utilizando como instrumento un cuestionario aplicado a los propietarios o administradores de las plantaciones bananeras seleccionadas. Este diagnóstico, permite hacer una valoración del escenario productivo.

\section{Instrumentos}

Se utilizó la encuesta, que es una técnica que consiste en obtener información acerca de una parte de la población o muestra, la misma que es proporcionada por ellos mismos, sobre opiniones, actitudes o sugerencias, por medio de un cuestionario con preguntas cerradas. Es impersonal porque el cuestionario no lleva el nombre ni otra identificación de la persona que lo responde, ya que no interesan esos datos.

Para la realización de esta encuesta se tomó.

- 10 fincas agremiadas en asociación economía popular solidaria.

- 10 fincas individuales de la economía familiar

20 fincas evaluadas en total

\section{Resultados}

- La finca tiene una persona encargada del área contable o financiera

Cuadro 1. La finca tiene una persona encargada del área contable o financiera

\begin{tabular}{|c|c|c|}
\hline $\begin{array}{c}\text { CONDICIONES } \\
\text { EVALUADAS }\end{array}$ & NUMERO DE FINCAS & PORCENTAJE \\
\hline SI & 6 & $30 \%$ \\
\hline NO & 14 & $70 \%$ \\
\hline
\end{tabular}

FUENTE: DATOS INVESTIGACIÓN

ELABORACIÓN: AUTORES

LA FINCA TIENE UNA PERSONA ENCARGADA DEL ÁREA

CONTABLE O FINANCIERA

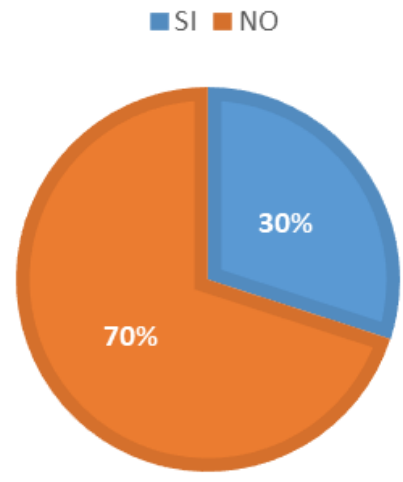

Figura 1. Resultados de datos del Cuadro $\mathrm{N}^{\circ} 1$. 


\section{Análisis}

De las 20 fincas encuestadas 14 corresponde al $70 \%$ manifestaron que no cuentan una persona encargada del área contable o financiera, solo 6 fincas que corresponde al $30 \%$ que si tienen este profesional el área financiera.

- La hacienda precisa de un profecional agrónomo para su administración

Cuadro 2. La hacienda precisa de un ingeniero agrónomo para su administración

\begin{tabular}{|c|c|c|}
\hline $\begin{array}{c}\text { CONDICIONES } \\
\text { EVALUADAS }\end{array}$ & NUMERO DE FINCAS & PORCENTAJE \\
\hline SI & 6 & $30 \%$ \\
\hline NO & 14 & $70 \%$ \\
\hline
\end{tabular}

FUENTE: DATOS INVESTIGACIÓN

ELABORACIÓN: AUTORES

\section{LA HACIENDA PRECISA DE UN INGENIERO AGRÓNOMO PARA SU ADMINISTRACIÓN}

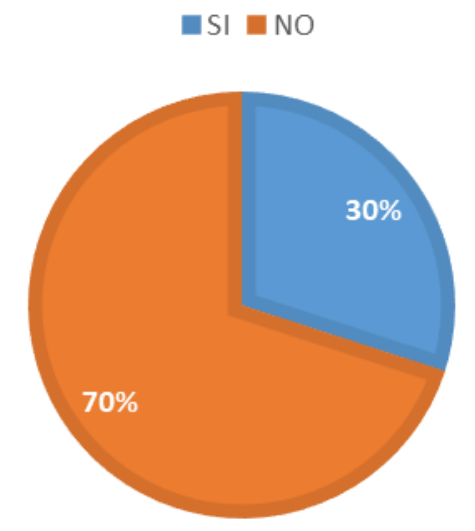

Figura 2. Resultados de datos del Cuadro $\mathrm{N}^{\circ} 2$.

\section{Análisis}

De las 20 fincas encuestadas 14 corresponde al $70 \%$ manifestaron que no cuentan con un profesional agrónomo para la administración de la finca, las 6 fincas restantes que corresponde al $30 \%$ que si tienen este profesional n el área de la administración. 
- Indique el sistema de siembra utilizado en la plantación

Cuadro 3. Indique el sistema de siembra utilizado en la plantación

\begin{tabular}{|c|c|c|}
\hline $\begin{array}{c}\text { CONDICIONES } \\
\text { EVALUADAS }\end{array}$ & NUMERO DE FINCAS & PORCENTAJE \\
\hline TRES BOLILLOS & 15 & $75 \%$ \\
\hline CUADRADO & 4 & $20 \%$ \\
\hline LINEAL & 1 & $5 \%$ \\
\hline
\end{tabular}

FUENTE: DATOS INVESTIGACIÓN

ELABORACIÓN: AUTORES

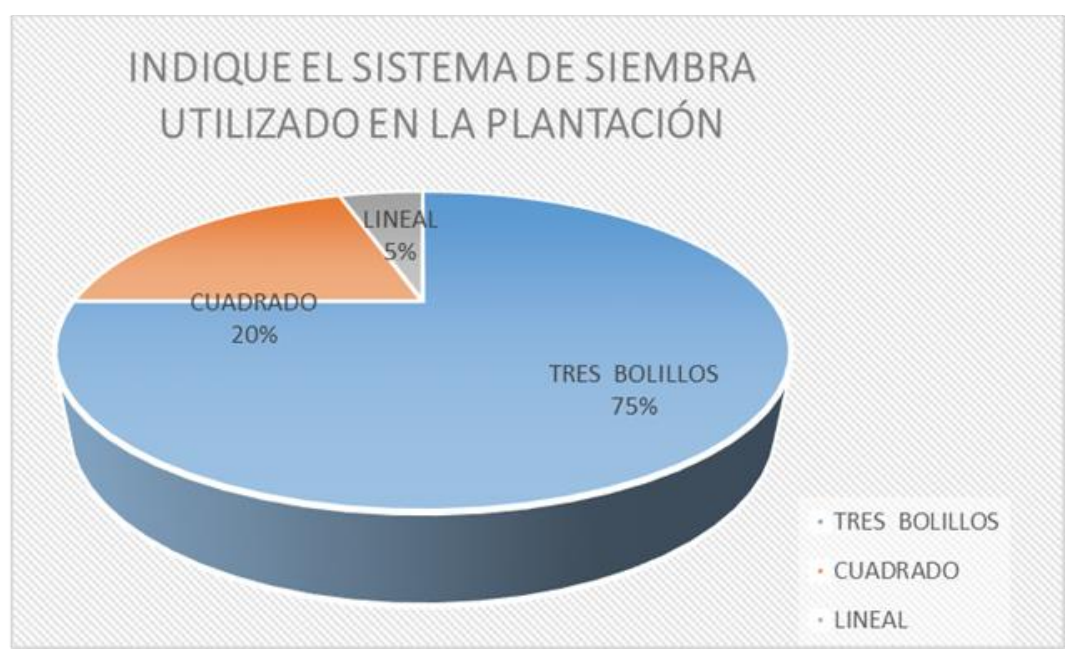

Figura 3. Resultados de datos del Cuadro $\mathrm{N}^{\circ} 3$.

\section{Análisis}

El 75\% de las haciendas que corresponde al número de 15 siembran en forma tres bolillos, el $20 \%$ es decir 4 fincas siembran la modalidad cuadrado y el $5 \%$ es decir 1 de las fincas 1 restante se inclina por la siembra lineal.

- Se ejecuta análisis de suelo para el desarrollo de programas de fertilización

Cuadro 4. Se ejecuta análisis de suelo para el desarrollo de programas de fertilización

\begin{tabular}{|c|c|c|}
\hline $\begin{array}{c}\text { CONDICIONES } \\
\text { EVALUADAS }\end{array}$ & NUMERO DE FINCAS & PORCENTAJE \\
\hline SI & 8 & $40 \%$ \\
\hline NO & 12 & $60 \%$ \\
\hline
\end{tabular}

FUENTE: DATOS INVESTIGACIÓN

ELABORACIÓN: AUTORES 


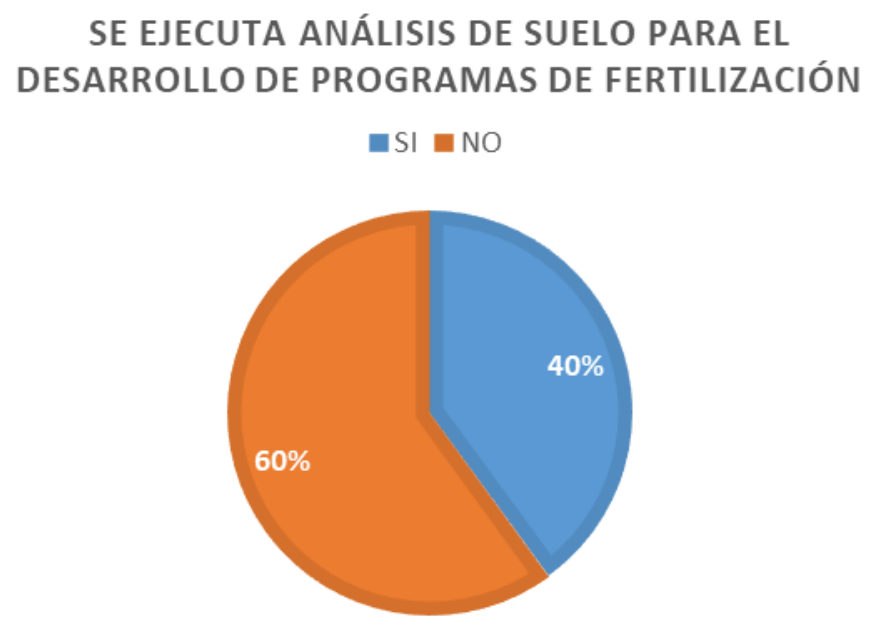

Figura 4. Resultados de datos del Cuadro $\mathrm{N}^{\circ} 4$.

\section{Análisis}

De las fincas encuestadas 12 corresponde al 60\% manifestaron que no ejecutan análisis de suelo para el desarrollo de programas de fertilización, las 8 fincas restantes que corresponde al $40 \%$ que si realizan el análisis.

- Se determina periódicamente el número de plantas por hectárea, mediante procesos de contaje de población.

Cuadro 5. Se determina periódicamente el número de plantas por hectárea, mediante procesos de contaje de población.

\begin{tabular}{|c|c|c|}
\hline $\begin{array}{c}\text { CONDICIONES } \\
\text { EVALUADAS }\end{array}$ & NUMERO DE FINCAS & PORCENTAJE \\
\hline SI & 13 & $65 \%$ \\
\hline NO & 7 & $35 \%$ \\
\hline
\end{tabular}

FUENTE: DATOS INVESTIGACIÓN

ELABORACIÓN: AUTORES 


\section{SE DETERMINA PERIÓDICAMENTE EL NÚMERO DE PLANTAS POR HECTÁREA, MEDIANTE PROCESOS DE CONTAJE DE POBLACIÓN.}

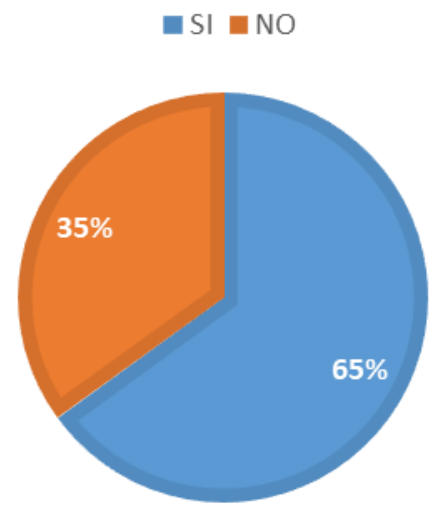

Figura 5. Resultados de datos del Cuadro $\mathrm{N}^{\circ} 5$.

\section{Análisis}

De las fincas encuestadas 13 corresponde al $65 \%$ manifestaron que determina periódicamente el número de plantas por hectárea, mediante procesos de contaje de población, mientras 7 agrícolas que corresponde el $5 \%$ no lo realizan el conteo.

- Qué sistemas de fertilización ejecutan en la hacienda bananera

Cuadro 6. Qué sistemas de fertilización ejecutan en la hacienda bananera

\begin{tabular}{|c|c|c|}
\hline $\begin{array}{c}\text { CONDICIONES } \\
\text { EVALUADAS }\end{array}$ & NUMERO DE FINCAS & PORCENTAJE \\
\hline MANUAL & 11 & $55 \%$ \\
\hline FERTIRIEGO & 2 & $10 \%$ \\
\hline $\begin{array}{c}\text { FOLIAR (COMBINADA } \\
\text { EN LA }\end{array}$ & 7 & $35 \%$ \\
FUMIGACION AÉREA) & & \\
\hline
\end{tabular}

FUENTE: DATOS INVESTIGACIÓN

ELABORACIÓN: AUTORES 


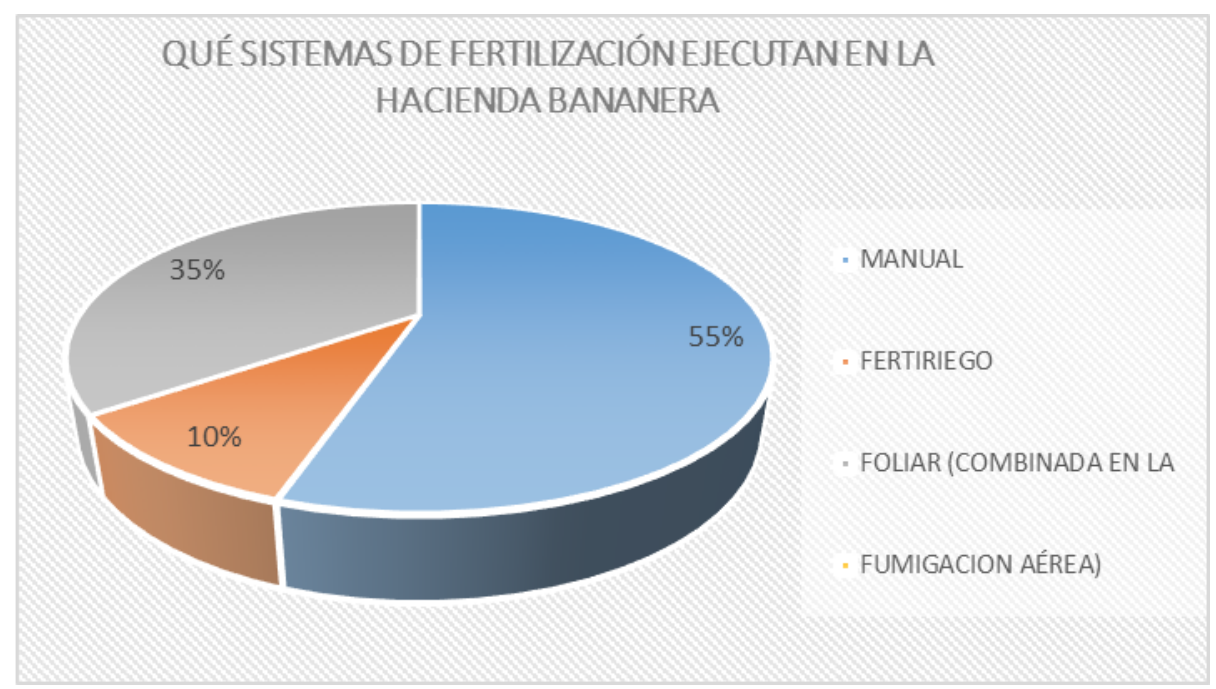

Figura 6. Resultados de datos del Cuadro $\mathrm{N}^{\circ} 6$.

\section{Análisis}

De las fincas encuestadas 13 corresponde al $65 \%$ manifestaron que determina periódicamente el número de plantas por hectárea, mediante procesos de contaje de población, mientras 7 que corresponde al que corresponde al $35 \%$ que no lo realizan.

- Establecen sistemas para monitoreo de la Sigatoka negra.

Cuadro 7. Establecen sistemas para monitoreo de la Sigatoka negra.

\begin{tabular}{|c|c|c|}
\hline $\begin{array}{c}\text { CONDICIONES } \\
\text { EVALUADAS }\end{array}$ & NUMERO DE FINCAS & PORCENTAJE \\
\hline PRE AVISO BIOLÓGICA & $\mathbf{4}$ & $\mathbf{2 0 \%}$ \\
\hline STOVER & $\mathbf{6}$ & $\mathbf{3 0 \%}$ \\
\hline EVALUACIÓN GENERAL & $\mathbf{1 0}$ & $\mathbf{5 0 \%}$ \\
\hline
\end{tabular}

FUENTE: DATOS INVESTIGACIÓN

ELABORACIÓN: AUTORES 


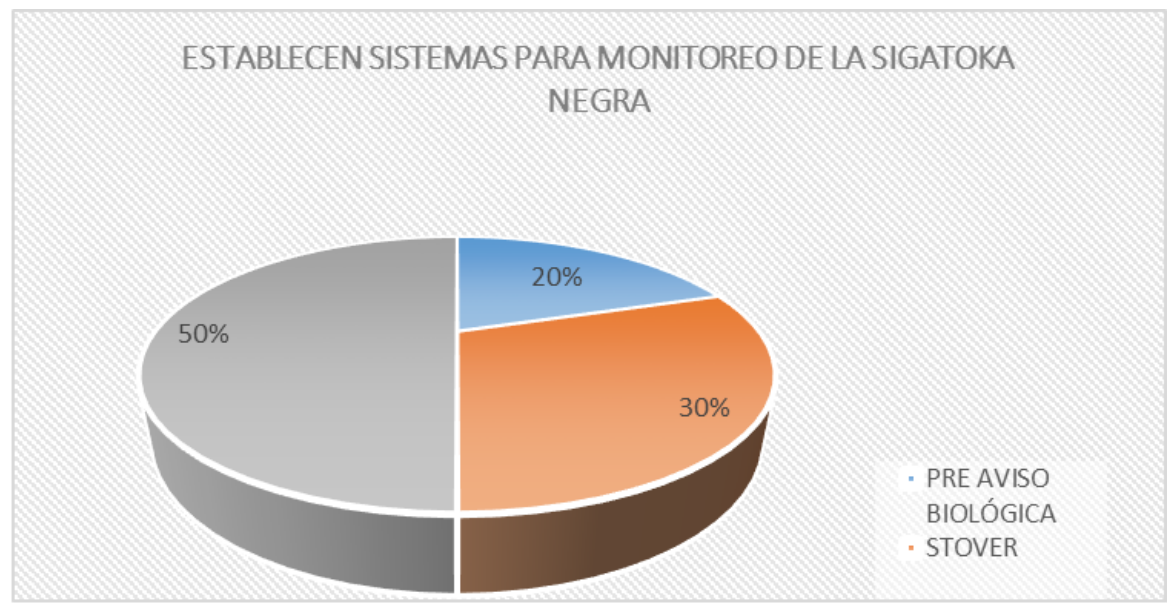

Figura 7. Resultados de datos del Cuadro $\mathrm{N}^{\circ} 7$.

\section{Análisis}

De las fincas 10 fincas corresponde al 50\% manifestaron que determina que establecen monitoreo evaluación general de la sigatoka negra periódicamente, 6 de estas que corresponde al que corresponde al 30\% utilizan STOVER. .4 que representa el 20\% utilizan PRE AVISO BIOLÓGICA.

- Qué sistema de fumigación tiene implementado en la finca

Cuadro 8. Qué sistema de fumigación tiene implementado en la finca

\begin{tabular}{|c|c|c|}
\hline $\begin{array}{c}\text { CONDICIONES } \\
\text { EVALUADAS }\end{array}$ & NUMERO DE FINCAS & PORCENTAJE \\
\hline FUMIGACION AÉREA & 10 & $50 \%$ \\
\hline NEBULIZACIÓN & 10 & $50 \%$ \\
\hline
\end{tabular}

FUENTE: DATOS INVESTIGACIÓN

ELABORACIÓN: AUTORES

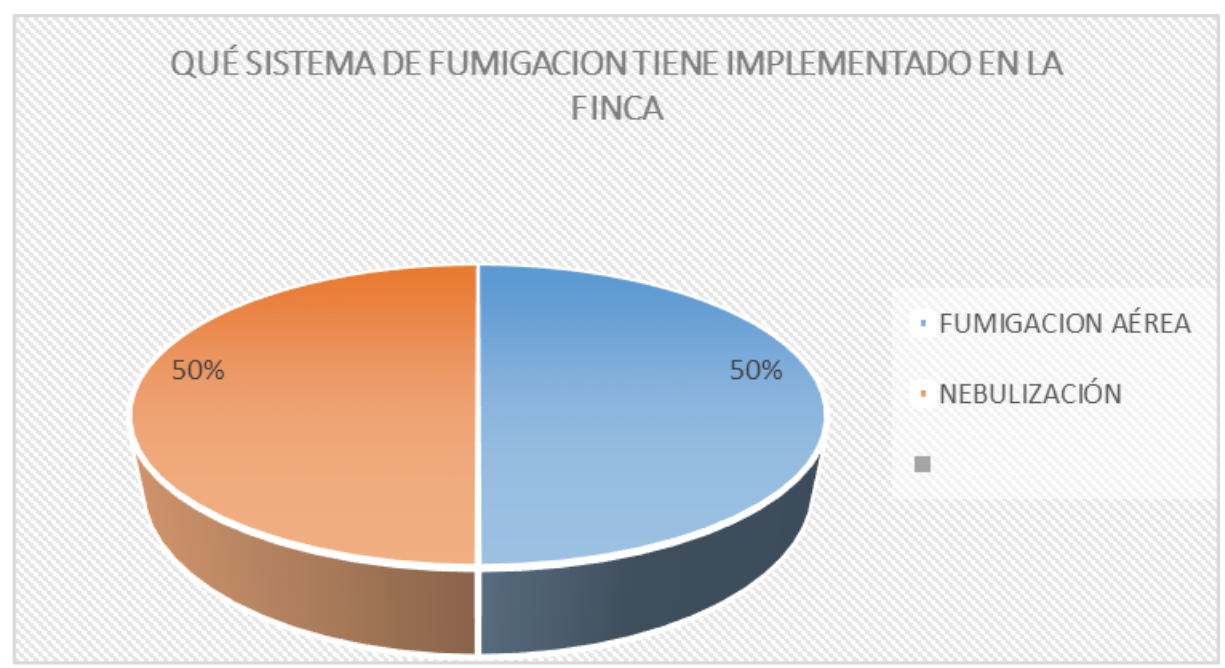

Figura 8. Resultados de datos del Cuadro $\mathrm{N}^{\circ} 8$. 


\section{Análisis}

De las 20 fincas encuestadas 10 fincas corresponden al $50 \%$ manifestaron determina que establecen la fumigación aérea mientras el otro $50 \%$ se realiza mediante nebulización

- Cuál es el sistema de cosecha utilizado en la finca

Cuadro 9. Cuál es el sistema de cosecha utilizado en la finca

\begin{tabular}{|c|c|c|}
\hline $\begin{array}{c}\text { CONDICIONES } \\
\text { EVALUADAS }\end{array}$ & NUMERO DE FINCAS & PORCENTAJE \\
\hline PODÓN & 12 & $60 \%$ \\
\hline ESCALERA ITALIANA & 2 & $10 \%$ \\
\hline GUADANA & 6 & $30 \%$ \\
\hline
\end{tabular}

FUENTE: DATOS INVESTIGACIÓN

ELABORACIÓN: AUTORES

\section{CUÁL ESEL SISTEMADE COSECHA UTILIZADO EN LA FINCA}

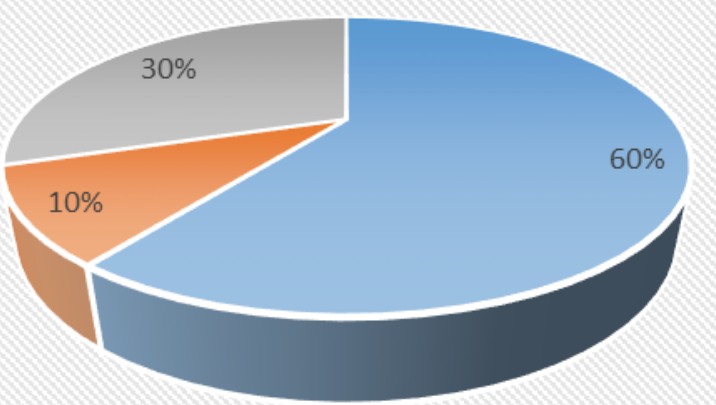

- PODÓN

ESCALERA ITALIANA

GUADANA

Figura 9. Resultados de datos del Cuadro $\mathrm{N}^{\circ} 9$.

\section{Análisis}

De las fincas 12 fincas corresponde al 60\% manifestaron que determina que utilizan el sistema de podón para la cosecha, 6 de estas que corresponde al que corresponde al $30 \%$ utilizan guadaña y 2 que representa el $10 \%$ utilizan escalera italiana.

- Qué sistema realizan para el pesaje del racimo al momento de evaluar la fruta en su ingreso a la empacadora. 
Cuadro 10. Conque sistema realizan el pesaje del racimo al momento de evaluar la fruta en su ingreso a la empacadora.

\begin{tabular}{|c|c|c|}
\hline $\begin{array}{c}\text { CONDICIONES } \\
\text { EVALUADAS }\end{array}$ & NUMERO DE FINCAS & PORCENTAJE \\
\hline BALANZA ANÁLOGA & 2 & $10 \%$ \\
\hline BALANZA DIGITAL 3 & 0 & $0 \%$ \\
\hline NO SE HACE & 18 & $90 \%$ \\
\hline
\end{tabular}

FUENTE: DATOS INVESTIGACIÓN

ELABORACIÓN: AUTORES

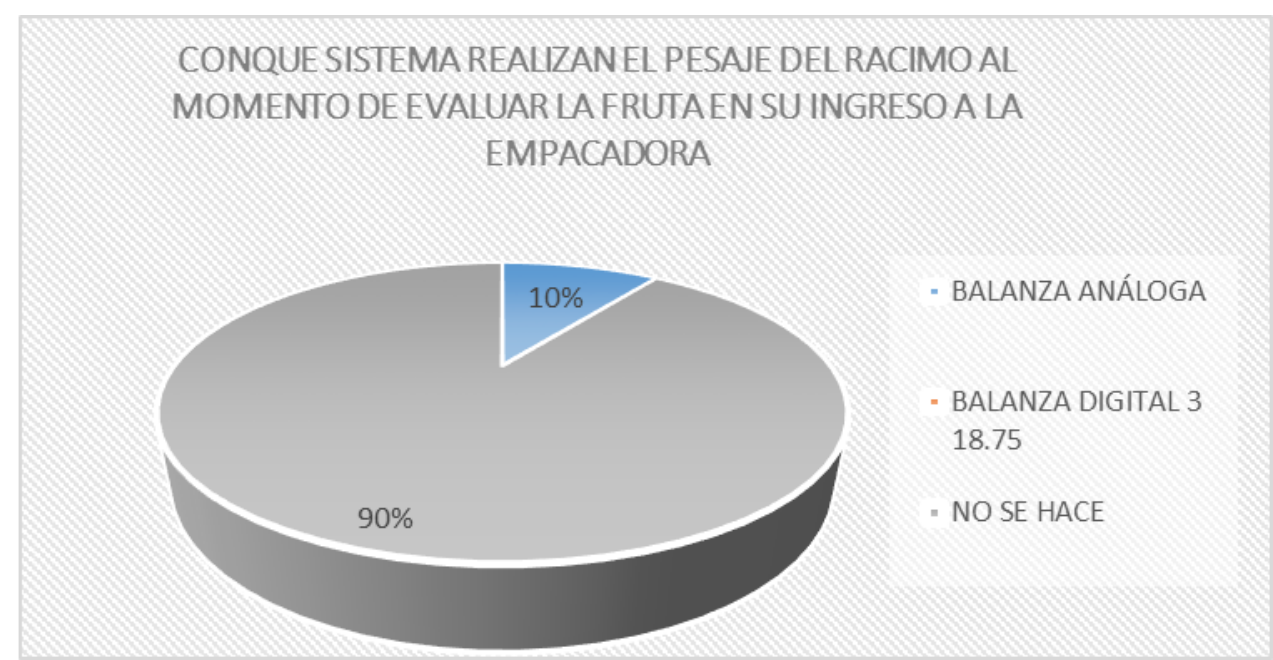

Figura 10. Resultados de datos del Cuadro $\mathrm{N}^{\circ} 10$.

\section{Análisis}

De las fincas 20 fincas, 18 que corresponde al $90 \%$ no realiza el pesaje de racimo, mientras que 2 que es el $10 \%$ lo ejecuta con balanza análoga.

- De qué manera se hace el proceso de aplicación de fungicida en la empacadora

Cuadro 11. De qué manera se hace el proceso de aplicación de fungicida en la empacadora

\begin{tabular}{|c|c|c|}
\hline $\begin{array}{c}\text { CONDICIONES } \\
\text { EVALUADAS }\end{array}$ & NUMERO DE FINCAS & PORCENTAJE \\
\hline MANUAL & 11 & $55 \%$ \\
\hline BOMBA DE MOCHILA & 9 & $45 \%$ \\
\hline APLICACIÓN & 0 & $0 \%$ \\
\hline
\end{tabular}

FUENTE: DATOS INVESTIGACIÓN

ELABORACIÓN: AUTORES 


\section{DE QUÉ MANERA SE HACE EL PROCESO DE APLICACIÓNDE \\ FUNGICIDAEN LAEMPACADORA}

- MANUAL

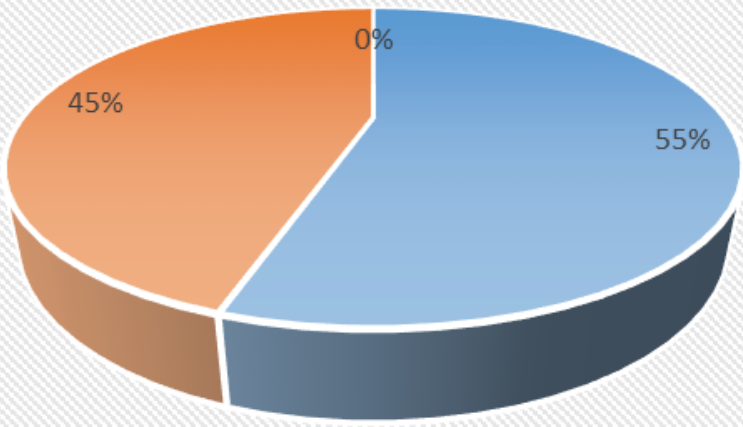

- BOMBA DE MOCHILA

APLICACIÓN

PRESURIZADA

Figura 11. Resultados de datos del Cuadro $\mathrm{N}^{\circ} 11$.

\section{Análisis}

De las fincas 20 fincas, 11 que corresponde al $55 \%$ realiza el proceso de aplicación de fungicida en la empacadora de manera manual, mientras 9 que representa el $45 \%$ por bomba de mochila.

- El número de cajas producidas por hectáreas de manera semanal se encuentra entre este parámetro.

Cuadro 12. El número de cajas producidas por hectáreas de manera semanal se encuentra entre este parámetro.

\begin{tabular}{|c|c|c|}
\hline $\begin{array}{c}\text { CONDICIONES } \\
\text { EVALUADAS }\end{array}$ & NUMERO DE FINCAS & PORCENTAJE \\
\hline $36-51$ & 12 & $60 \%$ \\
\hline $20-35$ & 6 & $30 \%$ \\
\hline $0-19$ & 2 & $10 \%$ \\
\hline
\end{tabular}

FUENTE: DATOS INVESTIGACIÓN

ELABORACIÓN: AUTORES 


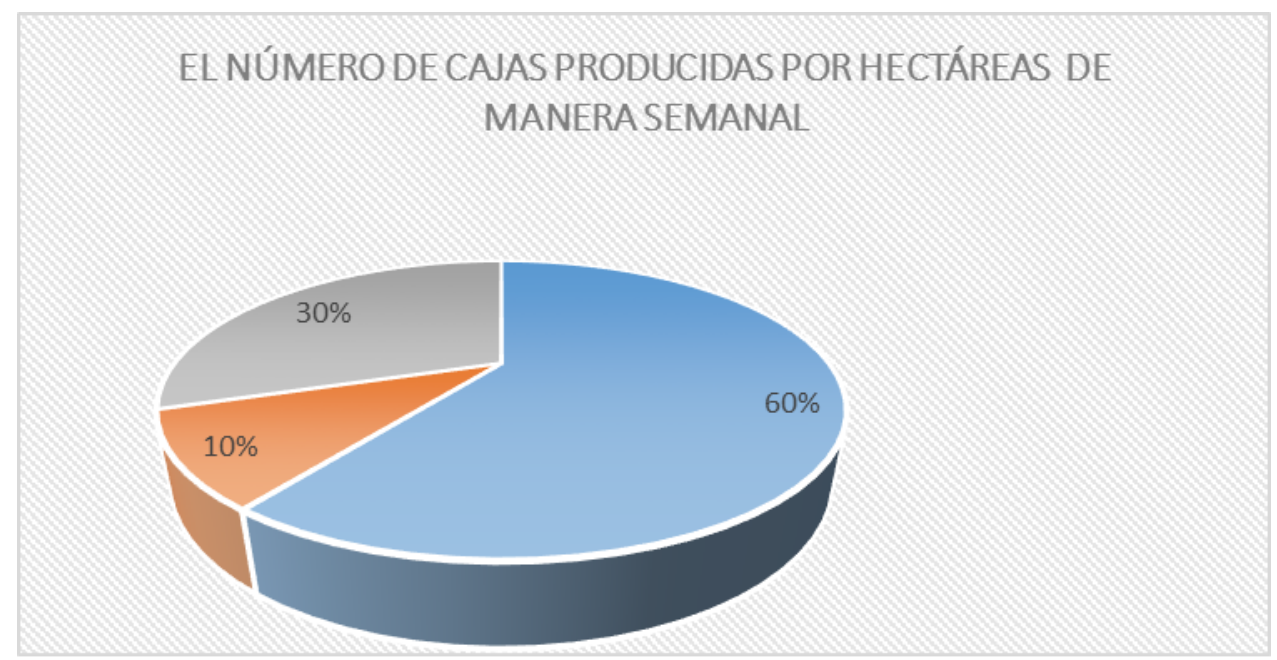

Figura 12. Resultados de datos del Cuadro $\mathrm{N}^{\circ} 12$.

\section{Análisis}

De las fincas 12 fincas corresponde al $60 \%$ manifestaron que determina entre 36 a 51 cajas producen de manera semanal, 6 de estas que corresponde al que corresponde al $30 \%$ tiene la productividad de 20 a 35 cajas y 2 que representa el $10 \%$ están en un rango 0 a 19 cajas de producción de manera semanal

- Los ingresos de ventas de banano semanales por hectárea en los siguientes parámetros.

Cuadro 13. Los ingresos de ventas del año anterior en los siguientes parámetros

\begin{tabular}{|c|c|c|}
\hline $\begin{array}{c}\text { CONDICIONES } \\
\text { EVALUADAS }\end{array}$ & NUMERO DE FINCAS & PORCENTAJE \\
\hline $144-208$ & 12 & $60 \%$ \\
\hline $80-140$ & 6 & $30 \%$ \\
\hline $0-76$ & 2 & $10 \%$ \\
\hline
\end{tabular}

FUENTE: DATOS INVESTIGACIÓN

ELABORACIÓN: AUTORES 


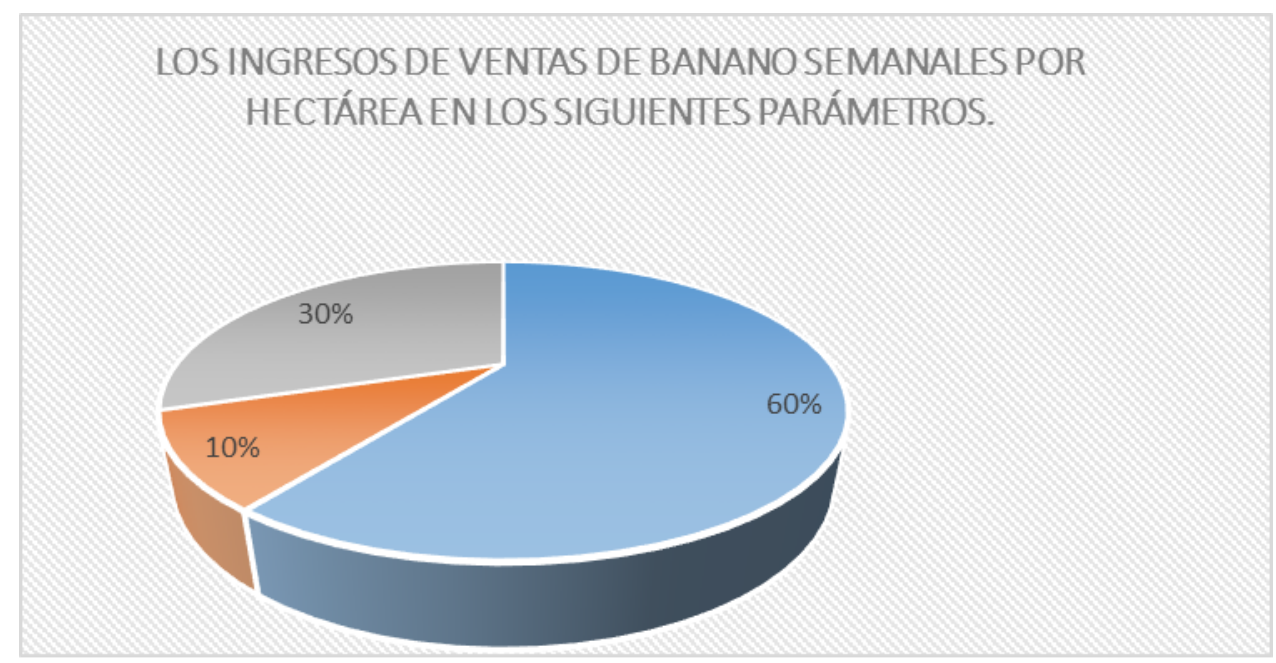

Figura 13. Resultados de datos del Cuadro $\mathrm{N}^{\circ} 12$.

\section{Análisis}

De las fincas encuestadas 12 que corresponde al $60 \%$ manifestaron tener un promedio de (144-208) dólares semanales, 6 agrícolas que corresponde al 30\% manifiestan tener ingresos de (80-140) dólares semanales, las 2 fincas restantes que representa el $10 \%$ están en un rango (0-76) cajas de producción de manera semanal.

\section{Conclusiones}

$>$ Se pudo determinar el que las fincas individuales de la economía familiar no poseen personal técnico en las áreas financieras y contables produciendo una incertidumbre en la producción en la parte financiera a diferencia de las fincas asociadas que tienen un profesional en estas áreas.

$>$ El manejo en campo y en la empacadora mejora el rendimiento productivo con la aplicación de tecnología y técnicas aplicadas con personal calificado en las fincas asociadas.

$>$ Los resultados de producción como de los de ingresos de ventas de las cajas de banano son marcadas las diferencias entre las fincas que fueron parte de este estudio, entre las haciendas individuales economía familiar y las asociadas fincas de la economía popular y solidaria

$>$ La incidencia del capital intelectual humano en el sistema de cultivo, producción, comercialización y administración, en las fincas asociadas que fueron parte de este estudio se observa el mejoramiento continuo en esta actividad económica. 


\section{References:}

1. El Foro Mundial Bananero. (15 de 06 de 2020). Organizaciòn de las Naciones Unidas para la alimentacion y la agricultura. Obtenido de http://www.fao.org/world-banana-forum/projects/covid-19/es/

2. Gaibor, J. (2018). Desarrollo de la agroindustria en la transformación de los sistemas productivos, modos de vida y la salud en la región agraria sur occidental del Ecuador. Quito: UASB-DIGITAL.

3. Acorbanec. (10 de Enero de 2019). Acorbanec Asociacionde comecialización y exportaciòn de banano. Obtenido de Acorbanec Asociacionde comecialización y exportaciòn de banano: http://www.acorbanec.com/noti70.html

4. Arbeláez, R. (2019). Incidencia de las exportaciones bananeras en la economia del ecuador periodo 2014-2017. Guayaquil: Universidad de Guayaquil.

5. Arguello, L., Purcachi, W., \& Pérez, M. (2019). La economía popular y solidaria en el desarrollo territorial. Análisis de las organizaciones del sector no financiero en la provincia de los Ríos-Ecuador. Dialnet, 16(53), 18.

6. Banco Central del Ecuador. (02 de Julio de 2019). Banco Central del Ecuador. Obtenido de Banco Central del Ecuador: https://www.bce.fin.ec/index.php/boletines-de-prensaarchivo/item/1182-la-econom\%C3\%ADa-ecuatorianacreci\%C3\%B3-06-en-el-primer-trimestre-de-2019

7. Banco Central del Ecuador. (17 de 01 de 2020). Banco Central del Ecuador. Obtenido de Banco Central del Ecuador: https://www.bce.fin.ec/index.php/boletines-de-prensaarchivo/item/1348-la-econom\%C3\%ADa-ecuatoriana-serecuperar\%C3\%A1-07-durante-el-2020

8. Camara Maritima del Ecuador. (07 de 09 de 2020). Camara Maritima del Ecuador. Obtenido de http://www.camae.org/exportaciones/rusiase-consolida-como-segundo-mejor-destino-del-banano-ecuatoriano-2/

9. Carrrillo, E. (2018). La influencia del sector bananero en la economía ecuatoriana, 2015-2016-2017. Guayaquil: universidad de Especialidades Espíritu Santo.

10. Coraggio, J. (2011). Economía social y solidaria; El trabajo antes que el capital. Quito: Abya-Yala.

11. Corpuagro. (28 de 05 de 2019). Banana exports. LaCompetitividad de las exportaciones de banano afectadas por un exceso de regulación, 10.

12. Criterios Digital. (18 de 09 de 2020). Criterios digital. Obtenido de Criterios digital: 
riteriosdigital.com/datos/deptoeconomicoycomercio/rusia-es-elsegundo-destino-de-exportacion-de-banano-ecuatoriano/

13. Drucker, P. F. (2002). La gerencia: Tareas, responsabilidades. Buenos Aires, Argentina: El Ateneo.

14. El Comercio. (04 de 01 de 2020). El Comercio. Obtenido de El Comercio: https://www.elcomercio.com/actualidad/exportacionesbanano-ecuador-crecieron-2019.html

15. El Telegrafo. (23 de 07 de 2020). El Telegrafo. Obtenido de El Telegrafo:

https://www.eltelegrafo.com.ec/noticias/economia/4/precio-bananocovid19

16. Fajardo, F. (2015). Gestión de recursos humanos en empresas agropecuarias. Buenos Aires: Pontificia universidad Católica Argentina.

17. Heredia, E. (2018). Evolución de la agricultura sostenible y agricultura convencional en el caso de cultivo de banano en ecuadorperiodo 2014 - 2017. Foz do Iguaçu: Universidad Federal da integracao Latino-Americana.

18. Instituto de Investigaciones Económicas y Políticas (IIEP) . (2017). Retos y Perspectivas del Desarrollo Económico en el Ecuador y América Latina. Guayaquil, Guayas, Ecuador.

19. Instituto Nacional de Investigaciones Agropecuaria. (2016). Fortalecimiento De Pequeños Productores De Banano Orgánico; Integración De Actores, Manejo Sostenible De Plagas Y Estrategias De Salud De Suelos. Quito: INIAP.

20. Jaramillo , E., Argüello, A., \& Advisor, P. (2020). Ecuador, líder en la producción de banano. Guayaquil: Ekos.

21. La Asociación de Exportadores de Banano de Ecuador (AEBE). (12 de 05 de 2020). banano en Ecuador. La Asociación de Exportadores de Banano de Ecuador (AEBE), 23.

22. León, L., \& Martínez, J. (2012). Caracterización y propuesta técnica acuacultura de las parroquias urbanas del sector Machala. Guayaquil: ESPOL.

23. Ministerio de Agricultura y Ganaderia. (03 de 10 de 2019). Ministerio de Agricultura y Ganaderia. Obtenido de Ministerio de Agricultura y Ganaderia: https://www.agricultura.gob.ec/sostenibilidad-garantizaproduccion-bananera-del-ecuador/

24. Ministerio de Comercio Exterior. (05 de 12 de 2017). Miniserio de Comercio Exterior. Obtenido de Miniserio de Comercio Exterior: https://www.produccion.gob.ec/wpcontent/uploads/2019/06/Informe-sector-bananero-espa\%C3\%B1ol04dic17.pdf 
25. Minsterio de Agricultura,ganaderia,acualcultura y pesca. (2019). El sector agropecuario ecuatoriano: análisis histórico y prospectiva a 2025. Quito: Ministerio de Agricultura, Ganadería, Acuacultura y Pesca 2019.

26. Monje, P. (2011). Economía solidaria, cooperativismo y descrentalizacion: la gestión social puesta en practica. Cuadernos EBAPE.BR, 9(3), 704-723.

27. Numbersnews. (29 de 03 de 2019). Numbersnews. Obtenido de Numbersnews: https://www.numbersnews.com/2019/03/ecuadorexportaciones-de-banano.html

28. Ollague , J., Capa, L., Novillo, E., Sanchez, T., \& Garcia, M. (2019). Variables sociales, económicas y productivas como referente de posicionamiento nacional de la provincia de El Oro, Ecuador. Espacios, 40(37), 13.

29. Pardo, C., \& Novillo, E. (12 de 2016). Pproceso de control de calidad para el banano de exportación en finca bananera. Observatorio Economía Latinoamericana., 1-15.

30. Pérez, O. (23 de 05 de 2016). Peoplenext. Obtenido de Gestión del talento humano en la empresa y su importancia: https://blog.peoplenext.com.mx/gestion-del-talento-humano-en-laempresa-y-su-importancia

31. Pino, S., Aguilar, H., Azuero, A., \& Sisalema, L. (2018). Aporte del sector agropecuario a la economía del Ecuador. Espacios, 7.

32. Revista Lideres. (02 de 10 de 2019). Revista Lideres. Obtenido de Revista Lideres: https://www.revistalideres.ec/lideres/mapamundibanano-ecuador-exportaciones-produccion.html

33. Sablich, C. (2012). Gestión de Recursos Humanos (GRH) Estratégica . Ica: Capacitación, Consultoría \& Asesoría .

34. Soler, R. (16 de 10 de 2017). Pymealdia. Obtenido de El recurso más importante de la empresa es el capital humano: https://www.pimealdia.org/es/el-recurs-mes-important-de-lempresaes-el-capital-huma/

35. Viteri, M. (23 de 02 de 2018). Economía ecuatoriana: de la producción agrícola al servicio. Espacios, 39(32), 1-30.

36. Zabala, V. (2019). Ecuador exporta casi el 25\% del banano del mundo. Ekos, 2. 